\title{
Editorial to the theme section on model-based engineering of smart systems
}

\author{
John Fitzgerald ${ }^{1} \cdot$ Fuyuki Ishikawa $^{2} \cdot$ Peter Gorm Larsen $^{3}$
}

Received: 11 September 2019 / Accepted: 16 September 2019 / Published online: 26 September 2019

(c) Springer-Verlag GmbH Germany, part of Springer Nature 2019

\section{Introduction}

The term 'smart' is widely applied to products and systems that are enabled by, and depend upon, computing and communication technology to analyse and respond to changing conditions in their environment. The category encompasses systems that range in scale from small devices to large-scale infrastructure, and in capability from those that undertake monitoring, control and optimisation to autonomous behaviour such as self-coordination with other systems in the environment, self-diagnosis and enhancement.

As reliance comes to be placed on the performance of such smart systems, the role of disciplined, model-based design grows in significance. At the same time, advances in the technologies employed in such systems present challenges to modelling and analytic capabilities. Smart systems are typically cyber-physical in character, demanding multidisciplinary design incorporating heterogeneous models and diverse simulation and analysis tools. The quality of human interaction, and hence the need for well-founded human-machine interface design, is increasingly critical. Furthermore, uncertainty about the behaviour of devices, systems and users in the environment, also becomes significant. All three areas stretch the state of the art and practice in model-based systems engineering.

The papers in this thematic section address some of these important and intriguing challenges. The section arises from

\footnotetext{
John Fitzgerald

john.fitzgerald@ncl.ac.uk

Fuyuki Ishikawa

f-ishikawa@nii.ac.jp

Peter Gorm Larsen

pgl@eng.au.dk

1 School of Computing, Newcastle University, Newcastle upon Tyne NE1 7RU, UK

2 National Institute of Informatics, Tokyo, Japan

3 DIGIT, Department of Engineering, Aarhus University, Aarhus, Denmark
}

work undertaken at the National Institute of Informatics Shonan Meeting on Model-based Design for Smart Products and Systems held on 4-7 December 2017 at the Shonan Village Center, Japan [1]. The meeting brought together scientists and engineers from a wide range of backgrounds to appraise the state of the art, develop a shared vision and prioritise challenges for future work at a range of technology levels. Following the workshop, the participants agreed to promote a theme issue of Software and System Modelling to include papers reporting results relevant to the challenges identified at the meeting.

\section{Selected papers}

Our selected papers show the potential of model-based methods in the design of smart systems. Although they each address fundamental challenges, they are also inspired by a diverse range of application domains: manufacturing, medical devices and the built environment. Among the papers, a common theme is the need for model-based engineering methods to promote dialogue between design disciplines, and between engineers and domain experts.

The engineering of smart cyber-physical systems entails the construction and analysis of models that utilise semantically diverse notations and abstractions, and that may be owned and managed by independent stakeholders. Verifying claims about system-level properties such as those relating to performance and dependability demands the integrated analysis of these models. In Early-Stage Analysis of Cyber-Physical Production Systems through Collaborative Modelling, Mihai Neghina, Constantin-Bala Zamfirescu and Ken Pierce show flexible methods for modelling and simulation in the design of such systems. They utilise the Functional Mockup Interface (FMI) standard to promote co-simulation, and in particular address the ways in which one can go about constructing multi-formalism models by first producing abstract co-models that are relatively homogeneous in character, allowing interaction protocols between constituent 
subsystems and key design parameters to be identified before effort is invested in heterogeneous modelling. Experience using the approach in a manufacturing environment helps to validate the findings.

As autonomy becomes more important in smart systems, the design of human-machine interfaces assumes vital significance. This requires interaction between relatively formal systems design processes and domain experts from different backgrounds. In A Framework for FMI-based Co-Simulation of Human-Machine Interfaces, Maurizio Palmieri, Cinzia Bernardeschi and Paolo Masci present a framework that allows formal methods experts to create interactive prototypes driven by formal models as a basis for this dialogue. A prototype based on similar co-simulation technology to that described by Neghina et al. is discussed and demonstrated using a case study based on a critical medical system.

The promotion of collaboration in design underpins the third paper in this collection. In Towards High-Level Fuzzy Control Specifications for Building Automation Systems, Juan C. Vidal, Paulo Carreira, Vasco Amaral, Joao Aguiam and João Sousa propose and demonstrate a domain-specific language for the increasingly significant application area of building automation systems. In this environment, where user perceptions are as important as sensor values, the language developed uses fuzzy temporal logic as an underpinning formalism. The utility of the language is validated with an empirical study.

These papers give an indication of the breadth and depth of advances required to promote methods and tools for the collaborative and cross-disciplinary development of smart cyber-physical systems. All three papers present tangible advances, and open up significant opportunities for future research, innovation and transfer of foundational research to industrial practice.

Acknowledgements We are profoundly grateful to the National Informatics Institute of Japan for supporting the Shonan Meeting series, which continues to provide inspiration to scientists and technologists worldwide. We thank the participants in our workshop for the stimulus to develop this theme issue. We are very grateful to the SoSyM editors and editorial office, notably Martin Schindler, for help and support in preparing this theme issue.

\section{Reference}

1. Ishikawa, F., Larsen, P.G., Fitzgerald, J.: Model-based design for smart products and systems: advanced capabilities and challenging applications. Technical Report No. 2017-18, Shonan Meeting, Japan, Dec 2017. ISSN 2186-7437

Publisher's Note Springer Nature remains neutral with regard to jurisdictional claims in published maps and institutional affiliations.

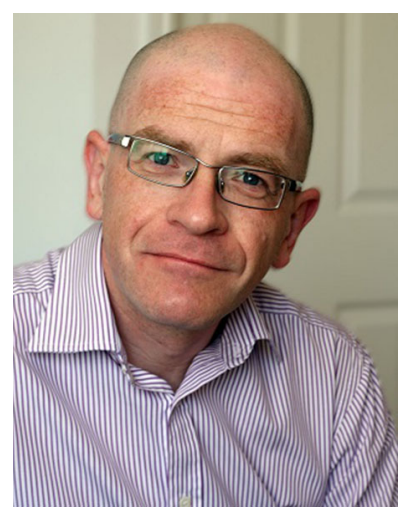

John Fitzgerald is a full professor in Computing Science at Newcastle University, UK, where he heads the School of Computing. His research is on modelbased methods and tools for system design and verification, and on their transfer to industry practice. Following his Ph.D. (1991) at Manchester University, UK, in modular proof techniques, he worked in and with the aerospace industry on formal methods in avionics design. He later led design and verification work in a successful start-up before returning to academia to lead research on formal model-based techniques for dependable systems-of-systems and cyber-physical systems.

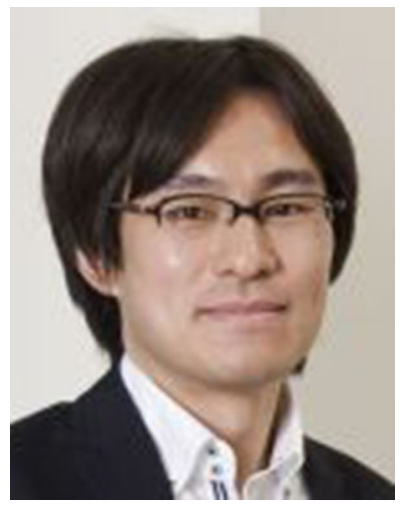

Fuyuki Ishikawa is Associate Professor at Information Systems Architecture Science Research Division, and also Deputy Director at GRACE Center, in National Institute of Informatics, Japan. His interests are in software engineering for dependability, including formal methods, testing, and optimisation, especially for smart and autonomous systems. His publications can be found in key journals and conferences, such as TSE, TPDS, TEVC, TSC, FM, ISSTA, WWW and ICSOC. His current focus is dependability of automotive systems and machine learningbased systems.

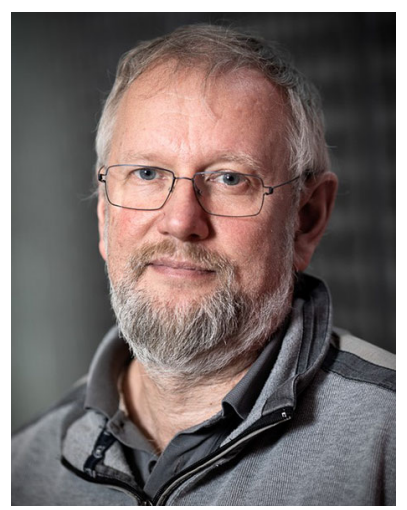

Peter Gorm Larsen is a full professor in the Department of Engineering at Aarhus University, where he leads the cyber-physical systems research group, the Centre for Digitalisation, Big Data, and Data Analytics (DIGIT), and the AU Centre for Digital Twins. $\mathrm{He}$ is also a visiting professor at York University and Newcastle University. After receiving his M.Sc. in Electronic Engineering at the Technical University of Denmark in 1988, he worked in industry before returning to complete a Ph.D. in 1995. As a development engineer and manager, he has given industrial courses worldwide, and has seen the VDM technology he developed through to commercial application in a wide range of areas including message processing, options trading and near-field communication firmware. 